\title{
Preimplantation genetic diagnosis for hereditary cancer syndrome: local experience
}

\author{
Vivian CY Lee ${ }^{1 *}$, FHKAM (Obstetrics and Gynaecology), Judy FC Chow², MPhil, Estella YL Lau', PhD, \\ Ava Kwong ${ }^{3}$, FRCS, FHKAM (Surgery), SY Leung ${ }^{4}$, FRCPath, FHKAM (Pathology), William SB Yeung'2, PhD, PC Ho², MD, \\ Ernest $\mathrm{HY} \mathrm{Ng}^{2}$, MD
}

\begin{abstract}
${ }^{1}$ Department of Obstetrics and Gynaecology, Queen Mary Hospital; ${ }^{2}$ Department of Obstetrics and Gynaecology, The University of Hong Kong; ${ }^{3}$ Hong Kong Hereditary Breast Cancer Family Registry; Division of Breast Surgery, Department of Surgery, Li Ka Shing Faculty of Medicine, The University of Hong Kong; ${ }^{4}$ Hereditary Gastrointestinal Cancer Genetic Diagnosis Laboratory, Department of Pathology, The University of Hong Kong, Queen Mary Hospital, Pokfulam, Hong Kong
\end{abstract}

Hong Kong Med J 2016;22:289-91

* Corresponding author: v200lee@hku.hk

DOI: 10.12809/hkmj144499

\section{Case reports}

We present three cases of preimplantation genetic diagnosis (PGD) performed for hereditary cancer syndromes at the Centre of Assisted Reproduction and Embryology, The University of Hong Kong, Queen Mary Hospital in Hong Kong.

\section{Case 1}

A 33-year-old woman was referred for consideration of PGD because she was a $B R C A 2$ gene mutation carrier. She had cancer of the right breast at the age of 24 years and underwent modified radical mastectomy with axillary dissection and immediate latissimus dorsi flap reconstruction. Adjuvant chemoradiotherapy was given and she was prescribed tamoxifen for 5 years after the operation. Her paternal grandmother had breast cancer diagnosed at the age of 60 years. Genetic screening was performed and confirmed the patient to be a BRCA2 mutation carrier. Her elder brother and her father underwent the spot test and were found to carry a BRCA2 mutation but her younger sister was not affected. Laparoscopic ovarian cystectomy was performed for a hyperechoic cyst noted over the right ovary, which was confirmed to be an endometriotic cyst. After a multidisciplinary meeting of clinical geneticists, breast surgeons, oncologists, gynaecologists, psychologists, nurses, and academics in the ethics department, followed by psychological assessment and also counselling, she was offered in-vitro fertilisation (IVF) and PGD. Her IVF and PGD cycle was performed in 2011, using an antagonist protocol with letrozole co-treatment. Fifteen oocytes were retrieved and 12 were fertilised following intracytoplasmic sperm injection (ICSI). Blastomere biopsy was performed on eight goodquality cleaving embryos and five were confirmed to be free of the BRCA2 mutation. Two unaffected blastocysts were transferred, resulting in a singleton pregnancy and one unaffected blastocyst was cryopreserved. She delivered a baby boy at term by caesarean section. Postnatal cord blood confirmed that the baby boy did not carry the BRCA2 mutation.

\section{Case 2}

A 33-year-old woman was referred for PGD because she was a carrier of FAP truncating germline mutation APC c.532-8G>A (NG_008481:g93262G>A) with a strong family history of colonic cancer. She underwent colonoscopy surveillance and more than 100 small colonic polyps were found. She was advised to have a prophylactic colectomy but was firm in her request to get pregnant with PGD treatment before the definitive treatment while fully understanding the risks of malignancy because of the delay in definitive treatment. She underwent an IVF cycle in 2012. Of 19 oocytes retrieved, 16 underwent ICSI. Fifteen were fertilised and 15 embryos were available for blastomere biopsy on day 3 . Five embryos were found without the FAP mutation and four goodquality blastocysts were cryopreserved due to the risk of ovarian hyperstimulation syndrome. She failed to conceive in two frozen embryo transfer (FET) cycles with one blastocyst replaced in each cycle. She subsequently underwent the last FET cycle with two blastocysts transferred, and a consequent singleton pregnancy. The pregnancy is 26 weeks' gestation at the time of writing. The couple refused an invasive prenatal test and requested postnatal cord blood confirmation.

\section{Case 3}

A 37-year-old patient was referred from a clinical geneticist for PGD as her husband was diagnosed to have neurofibromatosis type I and was a carrier of c.4495 C to T (p.Gln1499X) mutation in NF1 gene. The mutation is a nonsense mutation that changes the codon to a STOP codon. This mutation has not been reported to be associated with NF1, but such mutation is expected to result in a truncated protein product and is therefore very likely to be pathogenic. The couple were counselled accordingly and were very keen for PGD treatment. The woman underwent the first IVF cycle but only four oocytes 
were retrieved. Two mature oocytes were injected but only one was fertilised. The couple requested cryopreservation of the only embryo on day 2 . She underwent a second IVF cycle with five oocytes retrieved: four mature oocytes underwent ICSI and three were fertilised. The cryopreserved day- 2 embryo from the first cycle was thawed and cultured for 24 hours. A total of four embryos were available for embryo biopsy on day 3 and three were found to lack the NF1 mutation. Two blastocysts were transferred with a resulting singleton pregnancy. One surplus blastocyst was cryopreserved. After detailed counselling, the couple requested amniocentesis to confirm the PGD diagnosis and amniocentesis will be arranged at 16 to 18 weeks' gestation (at the time of writing the pregnancy is 14 weeks' gestation).

\section{Pre-preimplantation genetic diagnosis workup and preimplantation genetic diagnosis cycle}

\section{Case 1}

The exact genomic deletion breakpoints on the $B R C A 2$ gene were unknown when the patient first presented to us (the breakpoint was subsequently studied-c.7436_7805del [GeneBank U43746]) $)^{1}$ and the DNA of the patient's parents was unavailable. Therefore we tried to establish the haplotype around the $B R C A 2$ gene with the sibling DNA of the noncarrier sister and carrier brother. Nonetheless, the patient shared no common haplotype with her noncarrier sister and had exactly the same haplotype as her carrier brother around the BRCA2 gene. Finally, the high-risk haplotype was delineated by haplotype analysis of single sperms from her carrier brother. A PGD protocol was established that involved wholegenome amplification, ${ }^{2}$ linkage analysis with intragenic single-nucleotide polymorphism markers (rs1801406 and rs1799955) and flanking micro-satellite markers D13S289, D13S1698, D13S1701 and D13S171, located within $2 \mathrm{Mb}$ region flanking BRCA2 gene.

\section{Case 2}

Case 2 was a carrier of APC c.532-8G $>$ A (NG_008481:g93262G>A). The mutation was directly determined by minisequencing with SNaPshot Multiplex Kit (Applied Biosystems, Foster City, US). Linkage analysis was performed by the haplotyping $\operatorname{method}^{3}$ on a panel of 8 to 10 informative/partially informative microsatellite markers located within a $2 \mathrm{Mb}$ region flanking the $A P C$ gene. During the pre-PGD workup, neither sibling DNA nor offspring DNA was available to establish the highrisk haplotype, therefore high-risk haplotype was deduced from the genotype of embryos during PGD treatment cycles, by correlating the result of minisequencing and linkage analysis.

\section{Case 3}

The husband of case 3 was a carrier of mutation NF1c.4495C $>$ T (NM_000267). Mutation was directly determined by minisequencing and linkage analysis was performed on six informative/partially informative markers located within $2 \mathrm{Mb}$ region flanking NF1 gene. The high-risk haplotype was established by single-sperm haplotype analysis in the husband.

All PGD protocols were extensively validated against 40 single lymphocytes (20 maternal and 20 paternal) of the corresponding couples. During the PGD treatment cycle, all biopsied embryos resulted in a definitive diagnosis.

\section{Discussion}

The British Society of Gastroenterology recommends that all families with familial adenomatous polyposis (FAP) and Lynch syndrome should be screened in the context of a registry. A systematic review revealed that registration and screening resulted in a significant reduction in colorectal cancer incidence and mortality. ${ }^{4}$ With earlier detection of these cancer syndromes and a better surveillance system, patient survival is improved. ${ }^{5}$ As prenatal diagnosis is not a widely acceptable reproductive option, PGD to reduce the chance of having offspring with the same genetic predisposition to cancer is probably an attractive option after detailed counselling regarding the procedures and ethical concerns. It also avoids the need for termination. One recent study from the Netherlands showed that PGD is an acceptable choice for couples with hereditary breast and ovarian cancer (HBOC) ${ }^{6}$

In the first decade after the first published paper on PGD, the technique was used as an alternative to prenatal diagnosis for severe lethal inherited diseases. ${ }^{7}$ Indications for PGD have since been extended to adult-onset diseases such as Huntington chorea and spinocerebellar ataxia as well as diseases with incomplete penetrance such as hereditary cancer syndromes. It has been challenged ethically and the use of PGD in these indications was controversial, ${ }^{8}$ although both ESHRE (European Society of Human Reproduction and Embryology ${ }^{9}$ ) and HFEA (Human Fertilisation \& Embryology Authority; www.hfea. gov.uk) accepted these adult-onset and multifactorial diseases as indications for PGD.

Ovarian stimulation used for IVF treatment may trigger a high oestradiol concentration that may theoretically increase the risk of recurrence of hormone receptor-positive breast cancer. The use of letrozole to suppress the oestradiol concentration during IVF has been successful; some large case series have reported a comparable breast cancer recurrence rate in those who did and did not undergo IVF. ${ }^{10,11}$ A case-control study also revealed that even without letrozole, IVF treatment does not appear to 
increase the chance of breast cancer in $B R C A$ gene mutation carriers. $^{12}$

The use of prenatal invasive tests, such as chorionic villus sampling and amniocentesis, to confirm PGD results is controversial in adult-onset diseases with incomplete penetrance with the need for termination of pregnancy if the fetus is affected..$^{13}$ In our case series, only one couple out of three accepted the use of invasive prenatal tests and the possibility of termination. A recent study revealed that a proportion of couples with $\mathrm{HBOC}$ refused prenatal testing even following natural conception. ${ }^{6}$ In view of the growing number of requests for postnatal cord blood confirmation for these adultonset multifactorial diseases, its use in FAP families was discussed in our PGD ethics committee. This committee comprised reproductive medicine subspecialists, a clinical geneticist, maternal fetal medicine subspecialists, and laboratory in-charge. The pros and cons of postnatal testing were discussed. Since the risk of extra-colonic malignancies, such as hepatoblastoma, in FAP families is about 500 to 750 times that of the general population and the diagnosis is usually made before the age of 3 years, ${ }^{14}$ early diagnosis with postnatal confirmation of possible incorrect PGD diagnosis in order to have appropriate surveillance for these lethal malignancies would be considered worthwhile. A large case series also showed the importance of surveillance in paediatric FAP carriers of whom a considerable proportion with malignancies required treatment. ${ }^{15}$ The choice of using invasive prenatal procedures or postnatal cord blood testing to confirm a PGD diagnosis depends on discussion between the couple and the multidisciplinary team about the variable presentation of different syndromes.

Although PGD can be a practical and sound reproductive option for couples with hereditary cancer syndromes, awareness and knowledge of this technique is lacking even in prosperous developed countries such as the United States where PGD treatment is readily available. ${ }^{16,17}$ More information about PGD should be available to the general population, so that those who need this technique have access to this option and appropriate counselling.

\section{Conclusion}

The use of PGD is an alternative reproductive option for hereditary cancer syndromes. With good case selection, a multidisciplinary approach and support for the patient and family, this can be an acceptable option that takes account of the ethical concerns. More information about this technique should be provided to the general population and families with hereditary cancer syndromes.

\section{References}

1. Wang Q, Chow JF, Yeung WS, et al. Preimplantation genetic diagnosis using combined strategies on a breast cancer patient with a novel genomic deletion in BRCA2. J Assist Reprod Genet 2014;31:1719-26.

2. Chow JF, Yeung WS, Lau EY, et al. Singleton birth after preimplantation genetic diagnosis for Huntington disease using whole genome amplification. Fertil Steril 2009;92:828. e7-10.

3. Renwick P, Trussler J, Lashwood A, Braude P, Ogilvie CM. Preimplantation genetic haplotyping: 127 diagnostic cycles demonstrating a robust, efficient alternative to direct mutation testing on single cells. Reprod Biomed Online 2010;20:470-6.

4. Barrow P, Khan M, Lalloo F, Evans DG, Hill J. Systematic review of the impact of registration and screening on colorectal cancer incidence and mortality in familial adenomatous polyposis and Lynch syndrome. Br J Surg 2013;100:1719-31.

5. Vasen HF, Möslein G, Alonso A, et al. Guidelines for the clinical management of familial adenomatous polyposis (FAP). Gut 2008;57:704-13.

6. Derks-Smeets IA, Gietel-Habets JJ, Tibben A, et al. Decision-making on preimplantation genetic diagnosis and prenatal diagnosis: a challenge for couples with hereditary breast and ovarian cancer. Hum Reprod 2014;29:1103-12.

7. Handyside AH, Kontogianni EH, Hardy K, Winston R. Pregnancies from biopsied human preimplantation embryos sexed by Y-specific DNA amplification. Nature 1990;344:768-70.

8. De Wert G, Dondorp W, Shenfield F, et al. ESHRE task force on ethics and Law22: preimplantation genetic diagnosis. Hum Reprod 2014;29:1610-7.

9. Shenfield F, Pennings G, Devroey P, Sureau C, Tarlatzis B, Cohen J; ESHRE Ethics Task Force. Taskforce 5: preimplantation genetic diagnosis. Hum Reprod 2003;18:649-51.

10. Azim AA, Costantini-Ferrando M, Oktay K. Safety of fertility preservation by ovarian stimulation with letrozole and gonadotropins in patients with breast cancer: a prospective controlled study. J Clin Oncol 2008;26:2630-5.

11. Reddy J, Oktay K. Ovarian stimulation and fertility preservation with the use of aromatase inhibitors in women with breast cancer. Fertil Steril 2012;98:1363-9.

12. Kotsopoulos J, Librach CL, Lubinski J, et al. Infertility, treatment of infertility, and the risk of breast cancer among women with BRCA1 and BRCA2 mutations: a case-control study. Cancer Causes Control 2008;19:1111-9.

13. Moutou C, Gardes N, Nicod JC, Viville S. Strategies and outcomes of PGD of familial adenomatous polyposis. Mol Hum Reprod 2007;13:95-101.

14. Galiatsatos P, Foulkes WD. Familial adenomatous polyposis. Am J Gastroenterol 2006;101:385-98.

15. Munck A, Gargouri L, Alberti C, et al. Evaluation of guidelines for management of familial adenomatous polyposis in a multicenter pediatric cohort. J Pediatr Gastroenterol Nutr 2011;53:296-302.

16. Quinn GP, Vadaparampil ST, King LM, Miree CA, Friedman S. Conflict between values and technology: perceptions of preimplantation genetic diagnosis among women at increased risk for hereditary breast and ovarian cancer. Fam Cancer 2009;8:441-9.

17. Quinn GP, Vadaparampil ST, Miree CA, et al. High risk men's perceptions of pre-implantation genetic diagnosis for hereditary breast and ovarian cancer. Hum Reprod 2010;25:2543-50. 